Meta

Journal des traducteurs

Translators' Journal

\title{
Sur la notion de « terme »
}

\section{Marie-Claude L’Homme}

Volume 50, numéro 4, décembre 2005

Pour une traductologie proactive - Actes

For a Proactive Translatology - Proceedings

Por una traductología proactiva - Actas

URI : https://id.erudit.org/iderudit/012064ar

DOI : https://doi.org/10.7202/012064ar

Aller au sommaire du numéro

\section{Éditeur(s)}

Les Presses de l'Université de Montréal

ISSN

0026-0452 (imprimé)

1492-1421 (numérique)

Découvrir la revue

Citer cet article

L'Homme, M.-C. (2005). Sur la notion de « terme ». Meta, 50(4), 1112-1132.

https://doi.org/10.7202/012064ar

\section{Résumé de l'article}

Le présent article fait une synthèse de différents points de vue sur la notion de " terme " afin de répondre aux questions spécifiques qui se posent lors de l'élaboration d'un dictionnaire spécialisé. Notre étude des propositions faites dans des modèles théoriques de la terminologie et du traitement des termes dans les dictionnaires spécialisés existants révèle que les approches sont variées, souvent incompatibles et, qu'en définitive, certaines questions soulevées par la construction d'un dictionnaire restent sans réponse. Nous proposons donc une définition du terme en adéquation avec l'objectif applicatif annoncé et nous nous penchons sur ses conséquences méthodologiques. 


\title{
Sur la notion de «terme»
}

\author{
MARIE-CLAUDE L'HOMME \\ Université de Montréal, Montréal, Canada \\ mc.lhomme@umontreal.ca
}

\begin{abstract}
RÉSUMÉ
Le présent article fait une synthèse de différents points de vue sur la notion de «terme» afin de répondre aux questions spécifiques qui se posent lors de l'élaboration d'un dictionnaire spécialisé. Notre étude des propositions faites dans des modèles théoriques de la terminologie et du traitement des termes dans les dictionnaires spécialisés existants révèle que les approches sont variées, souvent incompatibles et, qu'en définitive, certaines questions soulevées par la construction d'un dictionnaire restent sans réponse. Nous proposons donc une définition du terme en adéquation avec l'objectif applicatif annoncé et nous nous penchons sur ses conséquences méthodologiques.
\end{abstract}

\begin{abstract}
This article examines and summarized different viewpoints on the notion of the "term" in order to answer the specific questions which arrise when compiling a specialized dictionary. We will give an overview of the answers found in current theoretical models as well as those that can be inferred from the examination of existing specialized dictionaries. Our overview reveals that approaches are varied and often incompatible. Furthermore, some questions remain unanswered when considering the problem of selecting terms in a dictionary. We will formulate our own definition which in is accourdance with the objectives we defined and we will examine its methodological consequences.
\end{abstract}

\section{MOTS-CLÉS/KEYWORDS}

terme, concept, sens, unité lexicale, structure terminologique

[...] the central object of terminology are terminological units (Cabré 2003: 183)

There is no fully operational definition of terms (Gaussier 2001: 168)

\section{Introduction}

Dans un contexte où les modèles terminologiques sont constamment remis en question (à un point où il apparaît presque suspect, dans certains cercles, de ne pas le faire), il semble opportun de revisiter les concepts fondamentaux auxquels ils font appel. Dans cet article, nous nous penchons sur la notion de "terme» qui, comme nous le verrons, est abordée sous des perspectives variées et souvent incompatibles.

Pour certains, le terme est l'objet central de la terminologie (par exemple, Cabré 2003; Kageura 2002); pour d'autres, le terme est une étiquette linguistique servant à matérialiser le véritable objet de la terminologie, à savoir le concept (Felber 1980; Wüster 1974). Cette distinction peut paraître réductrice, mais elle à l'origine de la plupart des antagonismes, du moins dans les cercles terminologiques (certains de ces antagonismes apparaîtront clairement plus loin). Par ailleurs, l'emploi même de terme est ambigu dans la littérature spécialisée. Pour les uns, il s'agit de la composante for- 
melle servant à communiquer un concept; pour les autres, il s'agit d'un signe linguistique, donc du résultat d'une association entre un sens et une forme. Enfin, de nombreux spécialistes envisagent le terme sans considérations théoriques véritables: pour eux, il s'agit d'une une entité formelle associée à un contenu informationnel (mot-clé pour la recherche d'information; étiquette associée au nœud d'une ontologie; entrée d'un index, etc.) (Ibekwe-San Juan et al. 2005).

Nous tentons ici de faire une synthèse de différents points de vue sur le terme en supposant que tous les spécialistes s'intéressent à des entités dotées d'un contenu et d'une forme. Les deux composantes ont des noms et des statuts différents d'un modèle terminologique à l'autre, mais elles sont toujours présentes. Par ailleurs, notre analyse sera guidée par une application spécifique, à savoir l'élaboration d'un dictionnaire spécialisé (l'application ainsi que les questions qu'elle soulève par rapport au terme font l'objet de la section 2). Nous croyons, comme Bourigault et Slodzian (1999), que la terminologie ne peut être appréhendée en faisant abstraction des objectifs de chacune de ses applications. Par conséquent, la notion de «terme» est toujours colorée par la perspective du spécialiste qui l'aborde. En outre, un ancrage dans la pratique nous apparaît essentiel à une époque où tout un secteur de la littérature terminologique est plus préoccupé par la théorisation que par le travail descriptif et l'étude de données.

L'article est organisé en cinq parties. Outre cette introduction et la conclusion, nous disons deux mots sur le contexte dans lequel nous situons notre étude (section 2). La section 3 examine les perspectives sur le terme adoptées par différents modèles théoriques et le traitement des termes dans les dictionnaires spécialisés; elle fait également une courte synthèse des enseignements que nous tirons de ces observations. Enfin, la section 4 présente une définition adaptée aux objectifs que nous avons fixés et montre son application concrète en insistant constamment sur ses conséquences méthodologiques.

\section{Le contexte}

La notion de «terme» est abordée ici dans un contexte applicatif spécifique, à savoir celui de l'élaboration d'un dictionnaire spécialisé. Nous travaillons présentement à la compilation d'un dictionnaire portant sur l'informatique et l'Internet - appelé DiCoInfo. Dictionnaire fondamental de l'informatique et de l'Internet - qui réunit des entrées décrivant les termes fondamentaux de deux domaines connexes ${ }^{1}$. Notre dictionnaire fournit de nombreux détails sur le sens et le fonctionnement linguistique des termes, mais évite de donner de longs développements encyclopédiques sur des concepts complexes.

Si la notion de "terme» est étudiée du point de vue de l'élaboration d'un dictionnaire spécialisé, deux premières questions très générales doivent être envisagées.

1. Quels sont les paramètres pouvant guider le terminologue lorsqu'il relève des termes dans les textes spécialisés?

Cette première question se pose lors de l'examen des textes spécialisés formant le corpus que le terminologue confectionne et qui sert de point de référence sur de nombreux aspects reliés à la description terminologique. Le terminologue, contrairement au lexicographe qui s'intéresse potentiellement à toutes les unités lexicales, doit faire un tri 
parmi les unités qui se succèdent dans un texte pour ne retenir que celles qui ont un lien avec le domaine qu'il est en train de décrire. Par ailleurs, le terminologue, comme il est rarement spécialiste du domaine sur lequel il se penche, peut difficilement faire appel à son intuition pour prendre des décisions et s'appuie davantage sur des données observables. Cette première question pose le problème de la définition du statut «terminologique» d'une unité linguistique.

2. Comment les unités considérées comme étant terminologiques doivent-elles être encodées dans le dictionnaire spécialisé?

Même si les décisions relatives à l'encodage doivent logiquement survenir à la suite de la sélection des termes, dans les faits, elles sont envisagées concurremment. Le terminologue sélectionnera des unités différentes en fonction du type d'encodage qu'il envisage. Cette seconde question soulève le problème des unités à inclure dans le dictionnaire ainsi que celle de leur organisation macro- et micro-structurelle.

À ces deux premières questions générales, se greffent un certain nombre de sousquestions sur lesquelles les modèles théoriques et les dictionnaires jettent des éclairages différents:

a. Les termes dénotent-ils des concepts ou ont-ils des sens (la réponse à cette question étant à l'origine de modèles d'encodage différents)?

b. Comment faut-il rendre compte des formes linguistiques qui dénotent plus d'un concept (ou qui ont plusieurs sens)?

c. Comment la variation (ou la synonymie) doit-elle être décrite?

d. Quelle(s) forme(s) ont les termes?

e. La structure terminologique doit-elle être décrite et, si oui, de quelle manière?

\section{3. Étude de quelques réponses}

Les réponses apportées aux questions soulevées dans la section qui précède sont étudiées en deux étapes. Nous procédons, en premier lieu, à l'examen de quelques modèles théoriques et tentons de résumer leur point de vue sur la notion de «terme». Nous nous tournons ensuite vers les dictionnaires spécialisés afin de mettre au jour les principes présidant au choix et au traitement des termes.

\subsection{Réponses apportées par les modèles théoriques}

Les modèles théoriques de la terminologie, souvent fondés sur des postulats et des objectifs qui leur sont propres, abordent la notion de «terme» sous des angles différents. Cette section fait un rapide survol des éléments permettant d'éclairer la notion de «terme» en les organisant autour de la distinction entre contenu et forme annoncée en introduction.

- Théorie générale de la terminologie (TGT) : La théorie générale de la terminologie (TGT) - attribuée à Wüster $(1968 ; 1980)$ et à ses disciples, souvent étiquetée terminologie classique ou terminologie traditionnelle - est à l'origine d'une conception du terme sur laquelle s'alignent encore la plupart des applications terminologiques ${ }^{2}$.

La TGT place le concept au cœur de la terminologie et considère que tout travail qui se réclame de la discipline doit s'appuyer sur la délimitation préalable qui en est faite. Le concept est envisagé comme un élément entrant dans une structure de connaissances et est appréhendé par une suite d'opérations de classement. Une définition servira à le fixer 
à l'intérieur du domaine de connaissances et à le baliser par rapport aux autres concepts avec lesquels il entretient un ensemble de relations logiques ou ontologiques.

La TGT préconise une démarche onomasiologique qui consiste à postuler d'abord l'existence du concept et découvrir, par la suite, les formes linguistiques servant à l'étiqueter. Le terminologue qui adhère à la TGT pourra faire un choix parmi les étiquettes d'un concept pour en élever une au statut de norme.

Depuis le milieu des années 1990, des voix se sont élevées contre cette optique de la terminologie qui, pour certains, est réductrice et ne tient pas compte de toute la complexité des applications modernes de la discipline. Les remises en question aboutissent parfois à la formulation de nouvelles approches. Celles que nous examinons dans cette section sont la socioterminologie (Gaudin 1993, 2003), la terminologie textuelle (Bourigault et Slodzian 1999), l'approche sociocognitive (Temmerman 2000) et la théorie communicative de la terminologie (Cabré 2003). Pour les uns, les principes wüstériens doivent être revus en profondeur (Gaudin 2003; Bourigault et Slodzian 1999; Temmerman 2000). Pour les autres, ces mêmes principes représentent des options distinctes dans une théorie plus étendue permettant d'accueillir des perspectives différentes sur la terminologie (Cabré 2003).

Nous limitons notre examen à ce que ces propositions disent sur la notion de «terme». On nous pardonnera les inévitables raccourcis que nous devons emprunter et qui occultent la complexité des modèles théoriques présentés.

- Socioterminologie: La socioterminologie (Gaudin 1993, 2003) est associée au cercle sociolinguistique de l'Université de Rouen et représente le premier mouvement d'opposition aux principes wüstériens. Les critiques sont nées de l'observation de la variation terminologique et de l'oral, mais touchent désormais de nombreux autres aspects théoriques de la terminologie.

Pour la socioterminologie, le concept n'est pas une entité figée et posée avant la découverte des désignations. Il se construit en discours où il subit toutes sortes de tensions et se modifie en fonction de variables sociales et historiques (Gaudin 2003). Le terminologue appréhende le concept à partir du stock lexical fourni par le discours. Cette démarche, clairement sémasiologique, est en porte-à-faux avec celle préconisée par la TGT. En outre, elle met également en évidence l'importance de la variation qui n'est plus envisagée comme un problème à résoudre, mais plutôt comme un phénomène qu'il importe de décrire.

Par ailleurs, la socioterminologie s'intéresse à la structure terminologique d'une manière qui diffère de celle proposée par la TGT. Bien que cette question ne semble pas complètement théorisée, le cercle de Rouen propose un modèle de dictionnaire dans lequel des relations lexicales (plutôt que conceptuelles) sont décrites (Gaudin et al. 2002) en s'appuyant sur une «sémantique relationnelle» (Gaudin 2003: 150).

- Terminologie textuelle: La terminologie textuelle (Bourigault et Slodzian 1999) est née de préoccupations applicatives, notamment l'interaction entre les travaux des terminologues et ceux d'informaticiens intéressés par le traitement des corpus spécialisés et l'élaboration d'ontologies ou autres formalismes destinés à représenter les connaissances.

La terminologique textuelle refuse, comme le fait la socioterminologie, le caractère fixe attribué a priori aux notions terminologiques. Elle envisage le terme comme un construit, c'est-à-dire comme le résultat d'une analyse faite par le terminologue qui prend en compte sa place dans un corpus, une validation par des experts et les objectifs d'une description. On remarque ici l'importance du discours, mise de l'avant par la socioterminologie; toutefois, l'ancrage concret est réalisé dans le corpus, matériau de base du terminologue. 
- Approche sociocognitive: L'approche sociocognitive (Temmerman 2000) remet en question de nombreux principes méthodologiques de la TGT. Elle souligne, comme le fait la socioterminologie, l'importance de la variation, moins dans sa dimension discursive, mais davantage dans sa contribution à la construction des concepts.

Dans l'approche sociocognitive, c'est surtout la nature du concept telle que l'envisage la TGT qui pose problème. Le concept n'est pas une entité figée mais plutôt quelque chose qui évolue et qui varie dans le temps. Temmerman préfere parler d'unité de compréhension (angl. unit of understanding) et propose de faire appel à la théorie du prototype pour en rendre compte.

- Théorie communicative de la terminologie (TCT): La TCT (Cabré 2003), comme nous l'avons signalé plus haut, se veut un modèle fédérateur dans lequel les différentes manières d'aborder la terminologie seraient prises en charge. Le noyau commun aux approches multiples doit toutefois être incarné par le terme. En TCT, la variété des accès possibles à l'unité terminologique est expliquée par un modèle dit des portes.

Pour Cabré (2003), la connaissance spécialisée est véhiculée par des formes linguistiques extrêmement diverses appelées unités de connaissance spécialisée (allant de symboles à des phrases entières en passant par les morphèmes dérivationnels, les unités terminologiques et les collocations). En TCT, les termes ne constituent qu'un moyen linguistique parmi d'autres pour exprimer la connaissance spécialisée et peuvent être abordés sous les angles cognitif, linguistique et communicatif (d'après une distinction proposée par Sager 1990). Les unités terminologiques ont toutefois un statut privilégié puisque, sous l'angle cognitif, il s'agit d'éléments qui entrent dans la structure conceptuelle du domaine; sous l'angle linguistique, il s'agit d'unités lexicales (Cabré 2003).

Puisqu'il s'agit d'unités lexicales, les termes peuvent coïncider dans leur forme avec des unités de langue générale (Cabré parle, dans ces cas, d'activation d'un sens spécialisé). Il peuvent appartenir aux parties du discours du verbe, du nom, de l'adjectif et de l'adverbe. En outre, les unités terminologiques peuvent correspondre à des structures plus complexes, c'est-à-dire à des syntagmes nominaux, adjectivaux, verbaux et adverbiaux.

\subsection{Réponses apportées par les dictionnaires spécialisés}

Après le rapide survol des propositions théoriques, nous examinons ce que les dictionnaires spécialisés 3 peuvent nous apprendre sur la notion de «terme». Comme il arrive souvent dans les applications, aucun dictionnaire ne constitue un reflet fidèle des principes théoriques évoqués ci-dessus ${ }^{4}$. Toutefois, il est possible de dégager deux tendances dominantes auxquelles ils souscrivent. Ces tendances expliquent comment le terminologue s'y prend pour décrire les termes et ont d'importantes implications méthodologiques et descriptives.

La plupart des dictionnaires spécialisés organisent les entrées autour de concepts, c'est-à-dire qu'ils considérent les termes comme étant l'expression linguistique de l'organisation des connaissances dans un domaine $e^{5}$, leur rôle consistant à rendre compte de cette organisation d'une manière ou d'une autre. Une forme linguistique (souvent un nom ou un syntagme nominal) devient terme lorsqu'on arrive à expliquer la place du concept qu'elle dénote dans le système conceptuel du domaine. Cette visée classificatrice de l'activité terminologique est résumée de la manière suivante par Rey:

Les systèmes terminologiques, formés de signes à fonction dénotative, référentielle et le plus souvent classificatrice, devront être organisés par l'ensemble des critères fondateurs 
de la classification des objets de connaissance: ils pourront être transformés en systèmes définitionnels explicitant ces critères (1976: 25).

La matérialisation des principes évoqués ci-dessus peut être illustrée par les descriptions proposées dans les banques de terminologie. Les fiches sont articulées autour de concepts fixés au moyen de définitions analytiques (une illustration de cette organisation est reproduite à la figure 1: il s'agit d'une partie de la fiche consacrée au concept «document hypertexte ...» extraite du Grand dictionnaire terminologique (GDT)).

FIGURE 1

\section{Extrait de la fiche document Web du GDT}

\section{document Web}

Document hypertexte diffusé dans le Web, qui forme un tout couvrant un sujet donné.

Syn. page Web document W3

pages Web

page W3

pages $W 3$

Les fiches ainsi présentées sont souvent décrites comme le résultat d'une démarche onomasiologique. Toutefois, dans les faits, le terminologue fait rarement de l'onomasiologie stricte, puisqu'il relève les formes linguistiques dans les textes. Mais il est vrai que l'exercice consistant à justifier la place d'un concept dans un système conceptuel (et, indirectement, des formes linguistiques servant à les désigner) impose un recul important sur les données linguistiques.

Dans ce type de description, il importe de distinguer formellement les concepts. L'application de ce principe dans les dictionnaires spécialisés mène à une conception des articles (ou des fiches) dans lesquelles les formes linguistiques dénotant plus d'un concept - appartenant ou non au même domaine - apparaissent sur des fiches différentes. Dans l'exemple de la figure 1, tous les termes sont des syntagmes nominaux et aucun n'apparaît sur une autre fiche dans le GDT. Toutefois, document et page sont associés à des concepts différents et font l'objet de descriptions distinctes (page, par exemple, peut désigner: 1 . une partie structurelle d'un texte; 2 . une partie de mémoire). L'organisation des entrées autour de concepts a une autre conséquence méthodologique. Toutes les formes linguistiques servant à nommer le même concept apparaîtront sur la même fiche. Ce principe est bien illustré à la figure 1: document Web, page Web, document W3 peuvent tous être expliqués au moyen de la même définition; ils sont donc alignés et traités comme des synonymes.

Un petit ensemble de dictionnaires spécialisés s'intéresse plutôt au sens (ou aux sens) que revêtent les formes linguistiques (généralement ramenées à des formes lexicales - nous reviendrons sur cette question plus loin). Le terminologue a un point de vue différent sur les unités et adopte une démarche sémasiologique pour les analyser. Dans cette optique, on hésitera à faire les distinctions et les regroupements de l'optique conceptuelle et dont témoigne la figure 1.

D'abord, page (signifiant «document hypertexte ...») peut s'utiliser seul et il ne s'agit pas simplement d'un raccourci anaphorique (page s'emploie aussi avec d'autres 
modificateurs - d'accueil, $\sim$ personnelle - et revêt dans ces syntagmes le même sens). Donc, il devrait être décrit comme un terme à part entière.

Comme dans la démarche conceptuelle qui distingue les concepts désignés par une même forme, le terminologue doit prendre des décisions sur le traitement des formes qui ont plusieurs sens. Si une forme lexicale est polysémique et que tous ou une partie de ses sens relèvent du domaine à l'étude, le terminologue pourra créer des articles distincts (modèle de la démarche conceptuelle) ou réunir ces sens dans le même article. Cette dernière méthode est illustrée par les deux articles de dictionnaire reproduits aux figures 2 et 3 .

FIGURE 2

Article symptomatique extraite de Manuila et al. (1994: 407).

symptomatique a (angl. symptomatic) 1) Qui se rapporte aux symptômes. 2) Qui vise à supprimer un ou plusieurs symptômes. Ex.: traitement symptomatique. 3) Qui survient en tant que symptôme particulier d'une affection. Ex. asthme symptomatique, névralgie symptomatique, maladie symptomatique.

FIGURE 3

Extrait de l'article investissement tirée de Binon et al. (2000: 334).

\section{INVESTISSEMENT}

1 un investissement

1.1 (emploi fréq. au pluriel) Opération économique ou financière par laquelle un agent économique (un particulier, une entreprise, un État - X) achète des biens de production, des valeurs mobilières ou un bien immobilier $(\mathrm{Y})$, ou consacre des sommes d'argent à la recherche ou à la formation $(\mathrm{Y})$ dans le but d'augmenter sa capacité de production ou ses revenus).

Ant.: un désinvestissement

1.2 (emploi fréq. au pluriel) Résultat d'une opération d'investissement (sens 1.1)

1.3 Opération par laquelle un agent économique (un particulier, une entreprise) met en réserve un bien de consommation durable pour le revendre ou le consommer plus tard. Syn. un placement

Le traitement de la synonymie variera également dans cette optique. Si on revient à l'exemple de la figure 1, le terminologue hésitera à faire les regroupements apparaissant dans la fiche de terminologie. S'il analyse document et page (seuls et non comme des têtes de syntagmes nominaux), le terminologue ne peut les envisager comme des synonymes exacts. Comme le montrent les exemples ci-dessous, les combinaisons admissibles pour page ne sont pas toutes valables pour document.

Vous pouvez consulter cette page.

Cette page a été visitée par un millier d'internautes.

Charger les pages des sites...

Vous pouvez consulter ce document.

?Ce document a été visité par un milliers d'internautes.

?Charger les documents des sites...

En outre, les liens synonymiques ne sont valables que pour une acception. Par exemple, dans l'entrée consacrée à investissement du Binon et al. (2000) (voir figure $3)$, le synonyme placement n'est donné que pour l'acception 1.3. 
Un deuxième aspect révélateur de l'optique adoptée par les dictionnaires spécialisés concerne la forme des entrées. Dans cette rubrique apparaît d'abord le problème du traitement des termes simples ou complexes. Doit-on faire le relevé systématique de l'ensemble des syntagmes nominaux dénotant un concept dans le domaine d'étude (car ils participent à la structuration de la connaissance) ou se limiter aux syntagmes dont le sens n'est pas compositionnel?

La plupart des dictionnaires spécialisés privilégient la première option. Par exemple, ils peuvent consacrer un article à fichier, mais créeront d'autres articles pour les syntagmes nominaux dont fichier est la tête ${ }^{7}$. Ce point de vue est adopté, notamment, par le GDT et TERMIUM qui consacrent plusieurs centaines de fiches aux termes dont une des composantes est est fichier. D'autres dictionnaires dresseront la liste des syntagmes nominaux comme sous-entrées dans l'article consacré à la tête, comme l'illustre la figure 4.

FIGURE 4

Liste de termes complexes en sous-entrée

FICHIER

$$
\begin{aligned}
& \sim \text { à accès aléatoire } \\
& \sim \text { à accès direct } \\
& \sim \text { de configuration } \\
& \sim \text { zippé }
\end{aligned}
$$

Le second problème que soulève la forme des termes concerne la partie du discours dans laquelle ils peuvent se réaliser. L'examen des dictionnaires spécialisés revèle que la partie du discours la plus largement représentée est celle du nom dans des proportions qui oscillent entre $84 \%$ et $98 \%$ (L'Homme 2003). Ce déséquilibre constitue un reflet de l'optique visant à décrire la structuration de la connaissances puisque les noms dénotant des entités sont les meilleurs candidats pour étiqueter un concept. La disproportion est amplifiée d'autant si le dictionnaire répertorie un nombre élevé de syntagmes nominaux.

Plus spécifiquement, le traitement des adjectifs varie d'un dictionnaire à l'autre et, souvent, au sein d'un même dictionnaire. Parfois, les adjectifs sont décrits dans un article qui leur est consacré, mais, le plus souvent, ils ne sont répertoriés que s'il apparaissent dans un syntagme nominal. Par exemple, exécutable peut figurer dans un dictionnaire uniquement s'il fait partie de syntagmes nominaux comme commande exécutable, fichier exécutable, module exécutable, programme exécutable (même si l'adjectif revêt toujours le même sens).

Comme les adjectifs, les verbes sont beaucoup moins présents dans les dictionnaires spécialisés. Toutefois, leur traitement ne s'aligne pas sur les mêmes principes puisqu'ils n'entrent pas dans la formation de syntagmes nominaux. Les dictionnaires relèvent les verbes qui sont propres à un domaine spécialisé (ex. déboguer) ou qui ont un sens distinct du sens qu'on leur donne généralement (ex. naviguer (dans l'Internet), piloter (un périphérique)). Ils hésiteront toutefois à inclure des verbes dont le sens ne se détache pas du sens qu'on leur attribue dans d'autres contextes. Par exemple, le traitement des verbes administrer (un réseau), exécuter (un programme), monter (un système de fichiers), déclarer (une variable) variera considérablement d'un dictionnaire spécialisé à l'autre. 
Enfin, le contenu des dictionnaires spécialisés reflète généralement une volonté de rendre compte d'une structure terminologique. Cette structure est rarement décrite de manière explicite, mais les liens sont perceptibles à toutes sortes de niveaux (par exemple, par un système de renvois ou dans la définition). La structure terminologique guide également le choix des termes.

Ici encore, deux points de vue s'opposent. Le dictionnaire qui adopte une démarche conceptuelle privilégie des relations hiérarchiques, comme l'hyperonymie et la méronymie. La synonymie envisagée est forcément exacte et l'antonymie est rarement considérée comme une relation valable (notamment, si le dictionnaire se concentre sur des termes de nature nominale). Le dictionnaire mettant le plus clairement en évidence les liens conceptuels est celui conçu par Wüster (1968) dont un article est reproduit à la figure 5 (les liens sont représentés par un système de renvois apparaissant dans la définition qui reportent l'utilisateur aux articles où sont décrits les termes).

FIGURE 5

Article machine(-outil) de précision Wüster (1968).

machine(-outil) de précision: Machine outil (810) destinée à usiner (808) des pièces (1390) avec une grande précision, c.-à-d. avec une tolérance (175) inférieure à $0.01 \mathrm{~mm}$.

Même s'il s'intéresse aux relations non hiérarchiques, le dictionnaire spécialisé à optique conceptuelle les relèvera dans la mesure où elles participent à la caractérisation des concepts. Par exemple, usiner est mis en évidence dans l'entrée reproduite à la figure 5 parce qu'il s'agit de l'activité typique rattachée à la machine-outil de précision et non parce que le verbe se combine de manière privilégiée avec ce nom.

Une démarche lexico-sémantique pourra s'intéresser à une multitude de relations lexicales (outre les relations signalées pour l'optique conceptuelle, on pourra tenir compte de relations actancielles, collocationnelles, etc.). Cette dernière observation est illustrée le mieux par le Binon et al. (2000) qui organise, dans une succession de rubriques différentes, les liens paradigmatiques et syntagmatiques que le terme décrit en entrée entretient avec d'autres unités lexicales. La figure 6 présente une partie des relations décrites dans ce dictionnaire pour investissement.

FIGURE 6

Partie de l'article investissement tirée de Binon et al. (2000: 334-337).

INVESTISSEMENT

\begin{tabular}{l|l}
1 un investissement & $\begin{array}{l}2 \text { un investisseur, } \\
4 \text { un désinvestissement } \\
\text { une investisseuse }\end{array}$ \\
4 le réinvestissement &
\end{tabular}

(définitions, Voir la figure 2) $\rightarrow$ société/entreprise - financement amortissement - épargne

+ adjectif 
TYPE D'INVESTISSEMENT (sens 1.1)

Un/des investissements + adjectif ... Un investissement initial (Syn. un investissement de départ, de base)

Un/des investissement(s) $\operatorname{direct}(\mathrm{s}) \ldots$

$\ldots$

CARACTÉRISATION DE L'INVESTISSEMENT (sens 1.1)

Un/de bon(s) investissement(s) ...

Un/des investissement(s) spéculatif(s) ...

Un/des investissement(s) rentable(s) ...

+ nom

(sens 1.1)

- Un société d'investissement ...

- Un club d'investissement ...

- Un plan d'investissement ...

+ verbe: qui fait quoi?

(sens 1.1)

\begin{tabular}{|c|c|c|c|}
\hline X & $\begin{array}{l}\checkmark \\
\times\end{array}$ & $\begin{array}{l}\text { prévoir un/des } \sim(\text { en/dans } \mathrm{Y}) \\
\downarrow \\
\text { réaliser un/des } \sim(\text { en/dans } \mathrm{Y}) \\
\ldots \\
\text { consentir un/des } \sim(\text { en/dans } \mathrm{Y}) \\
\text { effectuer un/des } \sim(\text { en/dans } \mathrm{Y}) \\
\text { procéder à un/des } \sim(\text { en/dans } \mathrm{Y})\end{array}$ & la réalisation d'(un) \\
\hline
\end{tabular}

3.3 Synthèse des réponses apportées par modèles théoriques

et les dictionnaires spécialisés

Le rapide survol proposé aux sections 3.1 et 3.2 montre que les les modèles théoriques et descriptifs de la terminologie proposent des solutions différentes aux questions soulevées par le terme. Il révèle également que, même si on envisage le terme dans son contenu, les perspectives sur la nature de ce contenu diffèrent sensiblement. Les différences sont suffisamment importantes pour forcer le terminologue à faire des choix parmi les options offertes et à en assumer les conséquences méthodologiques. L'examen du traitement des termes dans les dictionnaires spécialisés a d'ailleurs montré que la perspective adoptée a d'importance conséquences sur les descriptions produites.

Dans la plupart des approches décrites, le contenu du terme s'ancre dans la structuration des connaissances d'un domaine spécialisé. Il est intéressant de noter que les modèles théoriques ne remettent pas en cause de manière explicite la focalisation de la terminologie sur le concept et l'importance du système conceptuel. Ce sont surtout la nature du concept (notamment, son caractère immuable postulé par la TGT), la démarche onomasiologique et les objectifs de normalisation qui ont fait l'objet du plus grand nombre de critiques. Nous avons vu également que la plupart des dictionnaires s'aligne sur cette optique puisqu'ils s'attachent à décrire les termes sous leur angle conceptuel. 
L'optique conceptuelle (dans toutes ses déclinaisons) et les méthodologies qui en sont dérivées reproduit la démarche du spécialiste du domaine: donner des noms à des concepts (éventuellement les normaliser), structurer des connaissances, délimiter des concepts les uns par rapport aux autres, etc. Le terminologue s'aligne sur des modes d'appréhension des connaissances définies par les spécialistes ou, s'ils ne sont pas documentés, contribuera à les dévoiler. Pour construire une véritable représentation des connaissances, le terminologue doit forcément faire abstraction de la nature linguistique des termes. Il peut démarrer un travail à partir d'un corpus - c'est-à-dire puiser des formes linguistiques susceptibles d'étiqueter les concepts là où elles sont réalisées - mais il devra s'éloigner des observations qu'il en a tirées pour aboutir à une représentation compatible avec celle que produirait un spécialiste. Ce travail est donc mû par des impératifs extérieurs à la langue. Il a toutefois un certain nombre de conséquences qui se manifestent sur le plan linguistique: la démarche conceptuelle privilégie les termes de nature nominale, accepte un grand nombre de termes complexes et envisage la structure terminologique comme un ensemble de relations conceptuelles.

Même si on ne trouve pas de remises en cause affirmées de l'importance de la structuration des connaissances dans les modèles théoriques, certains d'entre eux font des aménagements afin de tenir compte de la nature linguistique du terme. L'« unité lexicale» est évoquée dans la TCT (Cabré 2003) et indirectement dans Gaudin (2003), qui parle d'un stock lexical. Toutefois, le terme continue d'y être présenté comme un moyen d'expression de concepts. Une démarche résolument lexico-sémantique n'a pas fait l'objet d'une théorisation en terminologie (du moins, pas officiellement, mais on en retrouve des éléments dans Béjoint et Thoiron 2000, Frawley 1988, Gaudin $2003^{8}$ et Kocourek 1991, entre autres). Cette absence de théorisation sur la démarche dans la littérature terminologique s'explique sans doute parce que les questions qui se posent trouve des réponses dans des modèles de sémantique lexicale.

Certains dictionnaires spécialisés se détachent plus radicalement de l'optique conceptuelle: ils envisagent les termes comme des unités lexicales et les appréhendent dans leur fonctionnement linguistique. Toutefois, il est intéressant de souligner que leurs concepteurs se réclament rarement de la terminologie ${ }^{9}$. Cette seconde optique mène à des descriptions qui constituent un reflet des observations faites sur un ensemble de données linguistiques et des interactions entretenues par les unités. Bien entendu, le terminologue prend des décisions au moment de rédiger des entrées de dictionnaires, mais le recul imposé par ce travail est moins important que celui nécessaire à une démarche conceptuelle. Cette démarche permet également de retenir des éléments descriptifs qui échappent à une perspective strictement conceptuelle: relations lexicales, variété des parties du discours, etc. Toutefois, elle ne peut postuler une structuration des connaissances a priori et on peut même douter que ses analyses mèneront à un modèle structurant au sens de la démarche conceptuelle. Elles peuvent donner lieu, en revanche, à une structuration lexicale.

\section{Le terme dans le DiCoInfo}

Nous avons affimé au début de la section 3.3 que le terminologue doit faire un choix parmi les options offertes et assumer ses conséquences méthodologiques. Le moment est venu de faire ce choix dans le contexte de notre dictionnaire de l'informatique et de l'Internet. 
Nous avons opté pour une optique dans laquelle le terme est envisagé comme une unité lexicale pour un certain nombre de raisons théoriques et méthodologiques que nous énumérons ci-dessous.

- L'appréhension d'un sens lexical peut s'appuyer sur l'étude de son environnement linguistique (qui fournira au terminologue des arguments pour valider la délimitation et la distinction des sens). La définition des concepts comme véhicules de la connaissance spécialisée suppose la sanction de spécialistes et, plus encore, qu'un consensus soit établi dans une communauté spécialisée. Si on admet ce qui vient d'être dit, la démarche conceptuelle est mieux servie par un spécialiste du domaine que par un spécialiste de la langue. Cette démarche est indispensable dans des entreprises où l'objectif consiste à expliquer des concepts: mais qui, mieux que le spécialiste, peut fournir ces explications?

- Les descriptions issues d'une démarche lexico-sémantique abordent et présentent les termes dans leur fonctionnement linguistique et admet donc plus facilement des unités lexicales appartenant à des parties du discours différentes.

- Les descriptions ne présupposent pas une structuration des connaissances a priori que seul un spécialiste véritable du domaine peut postuler. La structure lexicale peut être découverte au fur et à mesure de la description des termes.

- À notre avis, cette optique est plus compatible avec les méthodes actuelles de confection de dictionnaires (généraux et spécialisés) qui s'appuient principalement sur des corpus et qui adoptent une démarche sémasiologique. Nous dirons même qu'elle converge avec l'idée même qu'on se fait d'un dictionnaire. Accessoirement, l'optique que nous avons choisie a le mérite d'être plus proche de la formation actuelle des terminologues (dispensée dans des programmes de nature langagière, traduction et, parfois, linguistique). Elle fait d'abord appel à des connaissances en linguistique et en traitement de corpus; puis, à des connaissances des domaines étudiés (que le terminologue acquérera lors de la lecture du corpus et par la consultation de spécialistes).

Dans la suite de l'article, nous nous penchons sur les éléments d'une définition formelle du terme qui s'aligne sur les observations ci-dessus. Notre définition s'inspire d'éléments empruntés aux modèles théoriques et aux dictionnaires spécialisés présentés à la section 3, mais prend également en compte les objectifs spécifiques de notre entreprise lexicographique. Nous débuterons par des considérations méthodologiques avant de décrire la nature du terme telle que nous l'envisageons.

\subsection{Le terme défini dans un corpus}

Nous retenons d'abord l'idée de la terminologie textuelle selon laquelle le terme est un construit et qu'il est défini, entre autres, en fonction de la place qu'il occupe dans un corpus (Bourigault et Slodzian 1999) ou, de manière plus générale, le discours (Gaudin 2003). Ce premier postulat nous forcera à définir un corpus et à envisager une méthode pour évaluer la place des termes dans ce corpus.

La mise en forme du corpus succède à la définition des objectifs d'une description terminologique puisque le choix de textes doit être fait en adéquation avec ceux-ci. Les termes apparaissant dans le DiCoInfo sont d'abord extraits d'un ensemble de textes d'informatique français totalisant 1 million de mots. Les textes portent sur l'initiation à la micro-informatique, l'Internet, le logiciel, le matériel, la programmation, les réseaux et les systèmes d'exploitation. Le niveau de spécialisation des textes 
n'est pas homogène en ce sens que certains d'entre eux s'adressent à des experts, d'autres à non-experts. Toutefois, ils ont tous été rédigés par des spécialistes et ont écrits initialement en français (c'est-à-dire qu'il ne s'agit pas de traductions).

Nous déterminons le caractère spécifique des termes en nous appuyant en premier lieu sur un calcul automatique des spécificités mis au point par Drouin (2003) et implanté dans un logiciel appelé TermoStat. La technique met en opposition un corpus spécialisé (notre corpus d'informatique) et un corpus généraliste (le corpus Le Monde 2002) et fait émerger les unités dont la fréquence dans le corpus d'informatique est «anormale», c'est-à-dire proportionnellement beaucoup plus élevée dans le corpus spécialisé. Nous faisons l'hypothèse que la spécificité est un indice fort du statut terminologique des unités. Le tableau 1 montre les premières formes extraites par TermoStat ordonnées par ordre de spécificité décroissant.

Bien entendu, il s'agit d'une première approximation du statut terminologique puisque les formes spécifiques ne correspondent pas toutes à des termes (Lemay et al. 2005). Nous appliquons donc à la liste produite par le calcul automatique une série de critères lexico-sémantiques (ces critères sont énumérés à la section 4.2.1). Toutefois, la technique a le mérite d'offrir un ancrage dans le corpus.

Le corpus est également utilisé, d'une manière plus classique, à chacune des étapes de l'analyse des termes, à savoir, l'identification définitive du statut terminologique des unités, l'établissement de distinctions sémantiques et la recherche de liens sémantiques entre termes.

TABLEAU 1

Premières formes spécifiques du corpus d'informatique

\begin{tabular}{|c|c|c|c|}
\hline \multicolumn{4}{|c|}{ Vingt-cinq premières SP+ } \\
\hline Unité & Partie du discours & Fréquence brute & Valeur test \\
\hline fichier & SBC & 3956 & 360.825 \\
\hline commande & SBC & 1902 & 201.749 \\
\hline option & SBC & 1486 & 182.338 \\
\hline serveur & SBC & 1166 & 180.477 \\
\hline utilisateur & SBC & 1117 & 167.307 \\
\hline configuration & SBC & 845 & 162.82 \\
\hline utiliser & VB & 1996 & 161.788 \\
\hline répertoire & SBC & 1003 & 153.668 \\
\hline système & SBC & 2699 & 152.979 \\
\hline disquette & SBC & 609 & 148.431 \\
\hline ordinateur & SBC & 1283 & 140.484 \\
\hline touche & SBC & 855 & 138.648 \\
\hline logiciel & SBC & 1166 & 137.933 \\
\hline imprimante & SBC & 537 & 137.692 \\
\hline disque & SBC & 1093 & 129.889 \\
\hline mémoire & SBC & 1240 & 125.77 \\
\hline windows & SBP & 613 & 125.066 \\
\hline clavier & SBC & 579 & 122.24 \\
\hline caractère & SBC & 1096 & 118.081 \\
\hline recommander & ADJPAR & 516 & 117.004 \\
\hline
\end{tabular}




\begin{tabular}{|l|r|r|r|}
\hline linux & SBP & 442 & 116.261 \\
\hline bit & SBC & 382 & 110.72 \\
\hline paramètre & SBC & 455 & 109.856 \\
\hline interface & SBC & 412 & 109.64 \\
\hline permettre & VB & 2625 & 108,873 \\
\hline
\end{tabular}

\subsection{Le terme envisagé comme une unité lexicale}

Outre, l'ancrage dans le corpus, nous retenons également l'idée selon laquelle le terme est une unité lexicale (Cabré 2003) et que l'analyse terminologique s'appuie d'abord sur un stock lexical (Gaudin 2003). Toutefois, l'application de ce second postulat s'appuiera sur les observations faites dans les dictionnaires (section 3.2) plutôt que sur les modèles théoriques. Ce choix à un certain nombre de conséquences méthodologiques que nous énumérons dans les sous-sections qui suivent.

\subsubsection{Choix des termes}

Nous nous alignons pour définir la notion d'« unité lexicale» sur les définitions proposées par des lexicologues, notamment Cruse (1986) et Mel'čuk et al. (1995) pour qui les unités lexicales se distinguent formellement des autres unités linguistiques (morphèmes, syntagmes, phrases) par leur autonomie de fonctionnement dans la langue. Sur le plan sémantique, elles résultent de l'association d'une forme linguistique et d'un sens lexical (ce sens est appréhendé en observant l'ensemble des interactions qu'une forme lexicale entretient avec d'autres).

La particularité du terme, par rapport aux autres unités lexicales d'une langue, est d'avoir un sens spécialisé, c'est-à-dire un sens qui peut être mis en rapport avec un domaine de spécialité. Il n'est toutefois pas toujours exclusif au domaine étudié: certaines unités lexicales sont centrales dans un domaine mais sont largement utilisées ailleurs ou, encore, ne sont pas attachés à un domaine en particulier (par exemple, clavier apparaît dans tout dictionnaire d'informatique parce qu'il s'agit d'une partie importante de l'ordinateur; pourtant son sens en informatique ne se distingue pas nettement du sens qu'il véhicule dans d'autres situations; il s'agit toujours d'une partie d'appareil qui permet à un utilisateur d'entrer du texte, des chiffres ou des commandes).

La définition de "terme», contrairement à celle qui est donnée pour d'autres unités linguistiques, est donc relative. Elle dépend de la délimitation qu'on a faite d'un domaine spécialisé et les objectifs visés par une description terminologique.

Le terminologue doit donc établir un lien entre une unité lexicale et un domaine spécialisé. Cette démonstration s'appuiera forcément sur des connaissances du domaine (critère a. ci-dessous), mais elle peut être validées au moyen d'autres critères de nature lexico-sémantique (critères b., c. et d. ci-dessous) ${ }^{10}$.

a. L'unité lexicale a un sens lié à un domaine de spécialité; ce dernier est délimité au préalable pour un projet terminologique donné. Le lien avec le domaine de spécialité sera moins difficile à établir en ce qui concerne les noms dénotant des entités. Par exemple, les sens d'ordinateur, d'archive, de compilateur et de programme peuvent être mis en relation avec le domaine de l'informatique et nul ne contestera ce lien. Toutefois, le statut terminologique d'autres unités, notamment d'unités qui dénotent autre chose 
que des entités, sera plus difficile à démontrer. Les trois critères ci-dessous doivent être utilisés pour le définir.

b. La nature des actants sémantiques peut servir d'indice pour confirmer le sens spécialisé d'une unité lexicale de sens prédicatif. Si ses actants sont déjà admis comme termes en fonction du critère a., l'unité de sens prédicatif risque fort d'être spécialisée elle-même ${ }^{11}$. Les exemples ci-dessous montrent que les verbes adresser, héberger ainsi que l'adjectif compatible admettent deux termes comme actants, ce qui met en évidence le caractère potentiellement spécialisé de ces unités.

Ce système d'exploitation n'est soit pas conçu pour adresser plus de $1024 k$ de mémoire.

Le serveur qui héberge ce fichier coupe la communication.

Enfin, le moniteur doit être compatible avec la carte graphique déjà installée dans le système micro- informatique.

c. La parenté morphologique - nécessairement accompagnée d'une parenté sémantique - est un autre indice permettant de confirmer un sens spécialisé. Si des termes ont été retenus en vertu des critères a. et b., leurs dérivés sont forcément spécialisés. Par exemple, on admettra sans difficulté qu'archive est un terme dans le domaine de l'informatique puisqu'il signifie "Copie de données placée sur un support différent que celui sur lequel les données originales sont placées que l'utilisateur souhaite conserver». Il nous faudra donc admettre archiver, archivage, désarchiver (qui renvoient à différentes activités dans lesquelles un utilisateur place des fichiers dans un archive ou les extrait).

d. Toute autre relation paradigmatique, c'est-à-dire autre que morphologique, partagée par une unité lexicale avec un terme déjà admis en fonction des trois premiers critères, révèle un sens spécialisé. Par exemple, si interface est retenu en fonction du critère a., il faudra tenir compte de menu et de fenêtre qui sont des composantes faisant partie de l'interface. De même, si on admet qu'exécuter (une commande) est un terme, force sera d'admettre annuler qui est son antonyme.

Les critères ainsi formulés mettent en évidence que nous retenons des termes appartenant aux parties du discours du nom, de l'adjectif, du verbe et de l'adverbe (en -ment), point de vue compatible avec celui de Cabré (2003). Toutefois, notre analyse des termes comme unités lexicales a comme conséquence de nous amener à envisager surtout des termes graphiquement simples (ex. fichier, exécutable, visiter, numériquement). Les seuls termes complexes que nous retenons n'ont pas un sens compositionnel (ex. traitement de texte, système d'exploitation) ou, encore, sont composés d'éléments qui ne fonctionnent pas seuls dans le domaine de l'informatique (ex. fournisseur d'accès). Ainsi, nous retenons un petit nombre de locutions nominales (comme celles qui viennent d'être citées), de locutions verbales (ex. protéger en écriture) et de locutions prépositionnelles (ex. en arrière-plan, en ligne). Nous évitons également de traiter des syntagmes nominaux compositionnels (comme le font les dictionnaires adhérant à une optique conceptuelle: fichier exécutable ou fichier de configuration des erreurs du système) puisque les unités lexicales individuelles qui les composent font l'objet de descriptions à part entière.

\subsubsection{Distinctions sémantiques}

Comme nous l'avons signalé à la section 3.2, le terminologue doit forcément faire des distinctions sémantiques lorsqu'il aborde les formes dans les textes spécialisés. Certaines de ces distinctions serviront à écarter des sens qui ne sont pas reliés au 
domaine; d'autres auront pour but de séparer les acceptions spécialisées. Dans le DicoInfo, les distinctions sémantiques s'appuient sur nos connaissances du domaine étayées par des une série de tests lexico-sémantiques que nous énumérons ci-dessous.

a. La cooccurrence compatible et la cooccurrence différentielle (Mel'čuk et al. 1995: 64-66) permettent de déterminer si on a affaire au même sens ou à des sens différents en tentant de combiner l'unité à l'étude avec des cooccurrents différents. L'application de ces premiers critères permet de distinguer deux sens du verbe initialiser relevés dans un corpus d'informatique.

... les informations nécessaires pour initialiser l'ordinateur ... pour enregistrer les données sur une disquette verge, il faut d'abord l'initialiser ... *initialiser l'ordinateur et la disquette

b. La substitution par un synonyme (synonyme exact ou approximatif) vérifie la possibilité de substituer à un terme une autre unité lexicale dans un contexte donné. Ce critère permet de confirmer les deux sens d'accès illustrés dans les contextes suivants. Dans le premier exemple, on peut remplacer accès par connexion, alors que le second n'admet pas la substitution.

\section{accès à Internet connexion à Internet \\ accès à la mémoire $\quad{ }^{*}$ connexion à la mémoire}

c. La dérivation morphologique différentielle vérifie la possibilité de dégager des ensembles de dérivés morphologiques correspondant à des sens distincts. Ce critère permet de distinguer les deux sens d'adresse illustrés dans les exemples suivants. La première acception est liée aux dérivés adresser, adressable et adressage; ce n'est plus le cas pour la seconde.

$$
\begin{aligned}
& \text { adresse en mémoire } \\
& \text { adresser la mémoire } \\
& \text { adressage de la mémoire } \\
& \text { mémoire adressable } \\
& \text { adresse de site, de page Web } \\
& { }^{*} \text { adresser un site Web }
\end{aligned}
$$

d. La présence de liens paradigmatiques différentiels vérifie les oppositions et parentés sémantiques intervenant sur le plan paradigmatique (autres que les liens mis en évidence par les dérivés morphologiques). L'application de ce nouveau critère permet de distinguer deux des sens d'exécuter. Le premier entre dans le même paradigme que annuler, interrompre, abandonner, susprendre et reprendre, paradigme qui n'est plus valable pour le second.

$$
\begin{aligned}
& \text { exécuter une tâche } \\
& \text { annuler une tâche } \\
& \text { abandonner une tâche }
\end{aligned}
$$

L'application que quelques-uns de ces critères permettent de distinguer les sens de page rencontrés dans le corpus d'informatique (sens qu'illustrent les contextes cidessous). 
Le moteur cherche les pages contenant les mots clés.

Vous devez recharger en entier les pages des sites que vous visitez souvent.

La page d'accueil de ces moteurs de recherche offrent généralement ...

Ce sont les pages Web que vous voyez dans votre nabigateur.

Découper le programme en petites parties appelées pages ou segments.

Cette commande permet d'insérer une nouvelle page.

Les touches "page haut» et "page bas» affichent la page précédente ou suivante.

L'utilisation du critère $c$. montre que les occurrences de page apparaissant dans les trois derniers contextes peuvent être associés à des dérivés morphologiques (paginer, pagination, paginable), ce qui est impossible pour les quatre premiers. L'appel au critère d. révèle que page peut être rattaché à des holonymes différents: les 4 premiers contextes illustrent un sens de page qui peut être rattaché à site et portail; l'holonyme de page tel qu'illustré dans le 5e contexte est mémoire; celui de page apparaissant dans les deux derniers contextes est document ou texte. Enfin, le page des quatre premiers contextes peut être rattaché à un quasi-synonyme, c'est-à-dire document; le page de mémoire a un synonyme: segment; ces liens font défaut à l'acception de page illustrée dans le dernier contexte. Ces observations nous ameneront à distinguer trois sens pour page, chacun donnant lieu à une description différente.

\subsubsection{Structure terminologique}

Le choix d'envisager les termes comme des unités lexicales aboutit naturellement à une prise en compte de la structure lexicale à laquelle ils participent plutôt qu'à une description de la structure conceptuelle.

Dans le DiCoInfo, la structure lexicale sous-jacente au domaine de l'informatique et de l'Internet est mise en évidence explicitement par l'énumération de tous les termes partageant un lien de nature paradigmatique ou syntagmatique avec le terme décrit en entrée. De plus, une explication est donnée pour chaque paire de termes reliés.

Les relations sont dégagées à la suite de l'observation des interactions entretenues par un terme avec d'autres termes du domaine. Les liens sont observés dans le corpus ou, encore, apparaissent clairement lorsque de nouveaux termes sont décrits. Ce travail est entrepris afin de vérifier l'hypothèse selon laquelle la description des relations entretenues par des binômes contribuera à la découverte de la structure lexicale dans le domaine. La liste des relations ainsi que les explications s'inspirent fortement des fonctions lexicales de la lexicologie explicative et combinatoire (Mel'čuk et al. 19841999, 1995).

La figure 7 montre une partie de l'entrée PAGE 2. L'acception qui nous intéresse ici est celle reliée à l'entité placés dans le Web. La colonne de droite présente les termes qui ont un lien sémantique avec le terme décrit. Il sont accompagnés d'un numéro d'acception, ce qui permet de retrouver l'article correspondant à ce terme dans le dictionnaire (dans le site Internet où est placé une partie du contenu du dictionnaire, les entrées consacrées aux termes reliés sont accessibles par des hyperliens). La colonne de gauche présente l'explication du lien sémantique entre les termes. 
TABLEAU 2

Entrée simplifiée de PAGE 2 dans le DiCoInfo

\begin{tabular}{|c|c|}
\hline Actants et circonstants & Termes reliés \\
\hline Agent(s) & internaute 1 , visiteur 1 \\
\hline Créateur(s) & webmestre 1 \\
\hline Responsable(s) & hébergeur \\
\hline Patient(s) & information 1 , ressource 2 \\
\hline Destination(s) & World Wide Web 1 , toile 1 , Internet 1 , Web 1 \\
\hline Instrument(s) pour créer & éditeur 1 \\
\hline Instrument(s) pour utiliser & navigateur 1 ; fureteur 1 \\
\hline $\operatorname{Lieu}(s)$ & serveur 1 \\
\hline \multicolumn{2}{|l|}{ Sens voisins } \\
\hline Synonyme & $\sim$ Web \\
\hline \multicolumn{2}{|l|}{ Holonymes et méronymes } \\
\hline Tout & portail 1 , site 1 \\
\hline Élément constitutif & $\begin{array}{l}\text { texte } 1 \text {, image } 1 \text {, compteur } 2 \text {, formulaire } 1 \text {, } \\
\text { balise } 1\end{array}$ \\
\hline Division & en-tête de la $\sim$, titre de la $\sim$ \\
\hline \multicolumn{2}{|l|}{ Sorte de pages } \\
\hline Qui est créée à la demande de l'agent & $\sim$ dynamique 1 \\
\hline Qui ne change pas & $\sim$ statique 1 \\
\hline Qui change de contenu & $\sim$ évolutive 1 \\
\hline Qui est la première dans une succession & $\sim$ d'accueil \\
\hline Qui appartient à l'agent ou au créateur & $\sim$ personnelle \\
\hline Qui est associée à un format & $\sim$ HTML 1 \\
\hline \multicolumn{2}{|l|}{ Relations syntagmatiques } \\
\hline Dans & dans la $\sim$ \\
\hline Collocatifs reliés à la création & $\begin{array}{l}\text { créer } 1 \text { une } \sim(\text { création la d'une } \sim) \\
\text { éditer } 1 \text { une } \sim(\text { édition 1a d'une } \sim) \\
\text { générer } 1 \text { une } \sim(\text { génération la d'une } \sim)\end{array}$ \\
\hline $\begin{array}{l}\text { Collocatifs reliés à la publication dans } \\
\text { la destination }\end{array}$ & $\begin{array}{l}\text { mettre la } \sim \text { en ligne } 1(\text { mise en ligne } 1 \mathrm{a} \text { de la } \sim \text { ) } \\
\text { mettre la } \sim \text { à jour } 1(\text { mise à jour } 1 \mathrm{a} \text { de la } \sim \text { ) }\end{array}$ \\
\hline Collocatifs reliés à l'utilisation & $\begin{array}{l}\text { accéder } 2 \text { à la } \sim(\text { accès } 2 \mathrm{a} \text { à la } \sim, \sim \text { accessible }) \\
\text { charger } 1 \text { la } \sim(\text { chargement } 1 \text { a de la } \sim) \\
\text { recharger } 1 \text { la } \sim \\
\text { afficher } 2 \text { la } \sim(\text { affichage } 2 \mathrm{a} \text { de la } \sim) \\
\text { visualiser } 1 \text { la } \sim(\text { visualisation } 1 \mathrm{la} \text { de la } \sim) \\
\text { visiter } 1 \text { la } \sim(\text { visite 1a de la } \sim) \\
\text { consulter } 1 \text { la } \sim(\text { consultation } 1 \text { a de la } \sim, \sim \\
\text { consultable) }\end{array}$ \\
\hline $\begin{array}{l}\text { Collocatifs reliés à une utilisation } \\
\text { spécifique }\end{array}$ & $\begin{array}{l}\text { chercher } 1 \text { la } \sim \text {, rechercher } 1 \text { la } \sim \text { (recherche } 1 \mathrm{a} \\
\text { de la } \sim) \\
\text { indexer } 1 \text { la } \sim \text { (indexation } 1 \text { a de la } \sim, \\
\sim \text { indexée) }\end{array}$ \\
\hline
\end{tabular}




\section{Conclusion}

Nous nous sommes attachée, dans cet article, à présenter des perspectives théoriques et descriptives de la notion de "terme». Notre étude des propositions existantes a révélé que le terme, bien qu'il s'agisse pour plusieurs de l'objet central de la terminologie, reçoit des interprétations différentes conditionnées par des postulats théoriques ou des impératifs descriptifs divergents.

Nous avons limité notre réflexion à un cadre applicatif spécifique, à savoir l'élaboration d'un dictionnaire spécialisé. Ce travail a permis de montrer que le terminologue doit faire un choix entre deux conceptions centrales: 1. le terme comme étiquette de concept; 2 . le terme comme véhicule d'un sens spécialisé. Chacune des options a des conséquences méthodologiques et descriptives importances et mènent à des descriptions incompatibles. Nous avons opté pour une optique lexico-sémantique pour diverses raisons: elle offre un cadre permettant de valider les délimitations et les distinctions sémantiques; elle permet de mettre au jour la structure lexicale observable à l'intérieur d'un domaine spécialisé. Toutefois, cette démarche nous force à nous éloigner d'approches visant à révéler les structures de connaissances, mais, encore une fois, appartient-il au terminologue de faire ce travail?

Ces balises applicatives nous ont certes simplifié la tâche, mais nous croyons qu'il est extrêmement difficile, voire impossible, d'aborder le terme en faisant abstraction de l'application dans laquelle il est mis à contribution. La diversité des applications terminologiques (construction d'ontologies, élaboration de dictionnaires, documentation, traduction, etc.) et les postulats théoriques qu'elles appellent (description ou normalisation, cadre conceptuel ou lexico-sémantique, onomasiologie ou sémasiologie, etc.) mènent à des conceptions diversifiées de l'objet terminologique. Le terme donnera sans doute lieu à de nombreuses autres définitions et, à notre avis, il faut simplement accepter cette diversité (si elle peut être expliquée en fonction d'impératifs descriptifs) plutôt que de tenter de l'unifier. Les approches généralistes aboutiront à des définitions aux contours flous et à faible potentiel opératoire.

\section{NOTES}

1. Plus de 600 entrées peuvent être consultées à l'adresse suivante: olst.ling.umontreal.ca/dicoinfo/.

2. La conception de la TGT est reprise telle quelle ou avec des modulations mineures dans presque tous les manuels de terminologie (par exemple, Cabré 1992; Felber 1984; Rondeau 1984). En outre, elle est toujours dominante dans de nombreux programmes d'enseignement.

3. Ici, dictionnaire spécialisé inclut les banques de terminologie.

4. À l'exception du Dictionnaire de la machine-outil (Wüster 1968) qui a servi de base à l'élaboration de la TGT.

5. On reconnaît ici les principes fondamentaux de la TGT, mais également de la plupart des modèles théoriques présentés dans la section 4 , puisqu'on ne remet pas ouvertement la question de l'adéquation des descriptions terminologiques à la structuration des connaissances. C'est plutôt dans la manière dont on s'y prend qui est à l'origine des dissensions.

6. Soulignons que l'ordre d'apparition des formes linguistiques dans les articles ou les fiches est significatif en ce sens qu'il reflète souvent un choix fait par le terminologue. Certains dictionnaires et toutes les banques de terminologie iront plus loin en distinguant une vedette principale (considérée comme étant la forme correcte à utiliser) et d'autres formes linguistiques secondaires. Ces choix peuvent être associés aux objectifs de normalisation de la TGT.

7. Certains dictionnaires appliquent cette logique aux syntagmes verbaux et adjectivaux. Par exemple, dans le GDT, on trouve une fiche pour décrire le syntagme verbal convertir des données.

8. Gaudin (2003) aborde la terminographie en faisant appel à la sémantique lexicale. 
9. Par exemple, le le Binon et al. (2000) se présente comme un dictionnaire d'apprentissage. D'autres dictionnaires plutôt lexico-sémantiques sont décrits dans L'Homme (à paraître).

10. Ces critères ainsi que ceux qui valident des distinctions sémantiques sont également présentés dans L'Homme (2004).

11. Toutefois, pour être valable, ce second critère doit tenir compte d'un paramètre supplémentaire: l'unité de sens prédicatif n'a ce sens que lorsqu'elle est accompagnée des actants sémantiques spécialisés. Si l'unité de sens prédicatif véhicule le même sens avec des actants non spécialisés, alors elle n'est pas spécialisée elle-même.

\section{RÉFÉRENCES}

Béjoint, H. et P. Thoiron (éd.). (2000): Le sens en terminologie, Lyon, Presses universitaires de Lyon.

Binon, J., S. Verlinde, J. Van Dyck et A. Bertels (2000) : Dictionnaire d'apprentissage du français des affaires. Dictionnaire de compréhension et de production de la langue des affaires, Paris, Didier.

Bourigault, D. et M. Slodzian (1999): «Pour une terminologie textuelle», Terminologies nouvelles 19, p. 29-32.

CABré, M.T. (1992): La terminologia. La teoria, les mètodes, les aplicacions, Barcelona, Empùries.

CABré, M.T. (2003) : «Theories of Terminology: Their description, prescription and explanation », Terminology 9-2, p. 163-199.

Cruse, D.A. (1986) : Lexical Semantics, Cambridge, Cambridge University Press.

Drouin, P. (2003): «Term Extraction Using Non-technical Corpora as a Point of Leverage», Terminology 9-1, p. 99-115.

Felber, H. (1984): Terminology Manual, Vienna: Infoterm.

FraWley, W. (1988) : "New forms of Specialized Dictionaries », International Journal of Lexicography 1-3, p. 189-213.

Gaudin, F. (1993): Pour une socioterminologie: des problèmes sémantiques aux pratiques institutionnelles, Rouen, Publications de l'Université de Rouen.

Gaudin, F. (2003): Socioterminologie. Une approche sociolinguistique de la terminologie, Bruxelles, De Boeck/Duculot.

Gaudin, F., L. Guespin, A. Assal, V. Pierzo, M. Bouveret et K. Laval (2002): Lexpro, CDRom, La Maison du dictionnaire.

Gaussier, É. (2001): "General Considerations on Bilingual Term Extraction», In Bourigault, D., C. Jacquemin et M.C. L'Homme (eds.). Recent Advances in Computational Terminology, Amsterdam/Philadelphia, John Benjamins, p. 167-183.

Grand dictionnaire terminologique, GDT, Banque de terminologie de l'Office québécois de la langue française.

Kocourek, R. (1991): La langue française de la technique et de la science. Vers une linguistique de la langue savante, Wiesbaden, Oscar Brandstetter.

Ibekwe-San Juan, F., A. Condamines et M.T. Cabré (éd.) (2005) : Application-Driven Terminology Engineering. Special issue of Terminology 11-1.

Kageura, K. (2002): The Dynamics of Terminology. A theoretico-descriptive study of term formation and terminological concepts, Amsterdam/Philadelphia, John Benjamins.

L'Homme, M.C. (2003) : «Capturing the Lexical Structure in Special Subject Fields with Verbs and Verbal Derivatives: A model for specialized lexicography», International Journal of Lexicography 16-4, p. 403-422.

L'Hомме, M.C. (2004) : La terminologie: principes et techniques, Montréal, Presses de l'Université de Montréal.

L'Homme, M.C. (à paraître): «Using Explanatory and Combinatorial Lexicology to Describe Terms ", In Wanner, L. (éd.). Selected Lexical and Grammatical Topics in the Meaning-Text Theory. In Honour of Igor Mel'čuk, Amsterdam/Philadelphia, John Benjamins. 
Lemay, C., M.C. L'Homme et P. Drouin (2005): “Two Methods for Extracting 'Specific' Singleword Terms from Specialized Corpora", International Journal of Corpus Linguistics 10-2, p. 227-255.

Manuila, L., A Manuila et M. Nicoulin (1994): Dictionnaire médical, 6e édition, Paris, Masson.

MeL'ČUK, I. et al. (1984-1999): Dictionnaire explicatif et combinatoire du français contemporain. Recherches lexico-sémantiques 1-IV, Montréal, Les Presses de l'Université de Montréal.

Mel'čuk, I. A., Clas, A. et A. Polguère (1995): Introduction à la lexicologie explicative et combinatoire, Louvain-la-Neuve (Belgique), Duculot/Aupelf-UREF.

Rey, A. (1976): La terminologie: noms et notions, Paris, Presses universitaires de France.

Rondeau, G. (1984): Introduction à la terminologie, Montréal, Gaëtan Morin.

SAGER, J.C. (1990): A Practical Course in Terminology Processing, Amsterdam / Philadelphia, John Benjamins.

Temmerman, R. (2000): Towards New Ways of Terminological Description: The sociocognitive approach, Amsterdam/Philadelphia, John Benjamins.

Termium, Banque de terminologie du Bureau de la traduction, Gouvernement du Canada.

Wüster, E. (1968): The Machine Tool. An Interlingual Dictionary of Basic Concepts, London, Technical Press.

WÜsTER, E. (1974): Einführung in die allgemeine Terminologielehre und terminologische Lexicographie, Wien, Springer. 